\title{
AKUPUNKTUR AUCH IN COVID-19-ZEITEN EINE SICHERE SACHE!
}

\section{Liebe Kolleginnen, liebe Kollegen,}

die Akupunktur erfreut sich weiter steigender Beliebtheit. Jeder vierte Patient hat bereits Akupunkturleistungen in Anspruch genommen und zwei von vier Patienten erwägen Akupunktur als Therapieverfahren zu nutzen. Außerdem berichten Kollegen aus ihren Praxen seltener Terminabsagen im Lockdown bei Akupunktur und Störherddiagnostik als bei schulmedizinischen Terminen. Die Ausbildungssituation ist jedoch nach wie vor schwierig, da Akupunktur nur durch Präsenz in Praxiskursen wirklich erlernbar ist. Trotzdem kann mithilfe der WebSeminare der theoretische Anteil der Ausbildung gut und effektiv vermittelt werden, wie wir jetzt aus zahlreichen Veranstaltungen wissen. Die Resonanz der Kursteilnehmer ist überwältigend. Inzwischen haben wir auch bei der für uns zuständigen Bayerischen Landesärztekammer alle WebSeminare für die Weiterbildung anerkannt bekommen. Lediglich das „Gegenseitige Üben der Kursteilnehmer“ aus dem Kurs TCA 2,3 und 4 wurde mit 1 UE (= $45 \mathrm{~min}$ ) nicht akzeptiert. Dies stellt allerdings kein größeres Problem dar, da wir ab 2021 bei all unseren Hospitationskursen (Hands-on-Kursen) immer 45 Minuten eher beginnen und damit die fehlende Unterrichtseinheit automatisch nachgeholt wird. Der Hospitationskurs beginnt damit um 09:15 Uhr mit dem „Gegenseitigen Üben der Kursteilnehmer". Sie sollen in dieser Zeit die Akupunkturpunkte der Hauptmeridiane trainieren, in dem Sie sich mit einem Kollegen zusammentun. Der Referent steht für Fragen zur Verfügung. Da für die Zusatzbezeichnung Akupunktur fünf Hospitationskurse besucht werden müssen, besteht ausreichend Gelegenheit, die fehlenden UE im Rahmen dieser Kurse zu absolvieren. (https://www.akupunktur. de/aerzte/ausbildung-humanmedizin/ausbildungsleitfaden.html)
Fallkonferenzen | Da immer weniger Präsenzangebote in der Akupunktur bestehen, ist es für Kollegen schwer geworden, ihre Fallkonferenzen nachzuweisen. Bitte nutzen Sie unser Angebot an Hospitationskursen, Fortbildungskursen, Refresher-Kurs Garmisch (Mai 2021) und den Akupunkturkongress Timmendorf (Sept. 2021), da bei all diesen Präsenzveranstaltungen die Fallkonferenzen absolviert werden können. (https://www.akupunktur.de/ seminare.html)

Referentenschulung | Ende Januar trafen sich die Referenten unserer Akademie in Berlin zur jährlichen Referentenschulung. Angeregte Diskussionen zu Unterrichtsdidaktik und Lehrplan sowie zur Erweiterung der Lernangebote über unsere Homepage (Video-Tutorials, Podcast etc.) haben neben ungewöhnlichen Rahmenbedingungen wie „Essen nur am Platz mit großzügigem Hygieneabstand“ und „Essensbestellung über ein Lieferservice bei fehlender Hotelverpflegung “ dieses Treffen zu einer Schulung der besonderen Art werden lassen. Es zeigte sich, dass die direkte Begegnung und der direkte Austausch durch ein Zoom-Meeting nicht ersetzt werden können. Die lebendigen Diskussionen und Präsentationen neuer Diagnose- und Therapieansätze haben die Teilnehmer so begeistert, dass wir noch lange an diese schönen Stunden zurückdenken werden. Sicherlich werden unsere Mitglieder und Kursteilnehmer von den so erarbeiteten Ergebnissen im Laufe des Jahres profitieren. Lassen Sie sich überraschen!

Mit guten Wünschen für das Frühjahr,

Ihr

B. Ramme

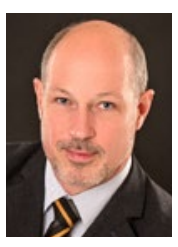

Dr. med. Bernd Ramme

1. Vorsitzender der DAA e.V.

Lerchenfeldstr. 20, D-80538 München

Tel. $+4989 / 8145252$

E-Mail des allg.Büros: kontakt@akupunktur.de, Internet:www.akupunktur.de 